Pacific

Journal of

Mathematics

THE NATURAL FILTRATIONS OF FINITE-DIMENSIONAL MODULAR LIE SUPERALGEBRAS OF WITT AND HAMILTONIAN TYPE

Keli Zheng, YongZheng Zhang And Wei Song 


\title{
THE NATURAL FILTRATIONS OF FINITE-DIMENSIONAL MODULAR LIE SUPERALGEBRAS OF WITT AND HAMILTONIAN TYPE
}

\author{
Keli Zheng, YongZheng Zhang And Wei Song
}

\begin{abstract}
We study the natural filtrations of the finite-dimensional modular Lie superalgebras $W(n, m)$ and $H(n, m)$. In particular, the natural filtrations which are invariant relative to the automorphisms of the Lie superalgebras are employed in order to characterize the Lie superalgebras themselves.
\end{abstract}

\section{Introduction}

In mathematics, a Lie superalgebra is a generalization of a Lie algebra including $\mathrm{a} \mathbb{Z}_{2}$-grading. Lie superalgebras are also important in theoretical physics where they are used to describe the mathematics of supersymmetry [Varadarajan 2004]. Although many structural features of Lie superalgebras over fields of characteristic zero (see [Kac 1977; Scheunert 1979]) are well understood, there seem to be very few general results on modular Lie superalgebras. In particular, the classification problem is still open for the finite-dimensional simple Lie superalgebras over fields of positive characteristic (see [Bouarroudj and Leites 2006; Zhang 1997] for example). The treatment of modular Lie superalgebras necessitates different techniques which are set forth in [Kochetkov and Leites 1992; Petrogradski 1992]. Elduque [2007] obtained two new simple modular Lie superalgebras. These Lie superalgebras share the property that their even parts are orthogonal Lie algebras and the odd parts are their spin modules. In [Zhao 2010] modular representations of basic classical Lie superalgebras were studied. The Lie superalgebras of Cartan type play an extremely important role in the study of modular Lie superalgebras. Recent works on them can be found in [Chen and Liu 2011; Yuan et al. 2011; Zhang and $\mathrm{Fu} 2002]$.

It is well known that filtration techniques are of great importance in the structure and the classification theories of Lie (super)algebras (see [Block and Wilson 1988; Strade 1993; Kac 1977; Scheunert 1979]). We know that the simple Lie

This work was supported by the National Natural Science Foundation of China (grant no. 11171055) and the Fundamental Research Funds for the Central Universities (no. 12SSXT139).

MSC2010: primary 17B40, 17B05; secondary 17B50, 17B70.

Keywords: modular Lie superalgebras, derivation superalgebras, natural filtrations. 
(super)algebras of Cartan type possess various natural filtration structures. For the filtration structures, the invariance may be used to make an insight for the intrinsic properties and the automorphism groups of those Lie (super)algebras. The natural filtrations of finite-dimensional modular Lie algebras of Cartan type were proved to be invariant in [Kac 1974; Kostrikin and Shafarevich 1969]. The finitedimensional simple modular Lie superalgebras $W, S$, and $H$ of Cartan type were defined in [Zhang 1997] and their natural filtrations were investigated in [Zhang and Fu 2002; Zhang and Nan 1998]. Recently, the natural filtrations of odd Hamiltonian superalgebras and special odd Hamiltonian superalgebras of formal vector fields were investigated in [Ren et al. 2012].

The finite-dimensional modular Lie superalgebras $W(n, m)$ and $H(n, m)$ were first introduced in [Awuti and Zhang 2008] and [Ren et al. 2011], respectively. In these papers, their derivation superalgebras were also determined. The starting point of our studies is the investigation of the ad-nilpotent elements of $W(n, m)$. Then the natural filtration of $W(n, m)$ is proved to be invariant by the determined ad-nilpotent elements. In the case of $H(n, m)$, the invariance of the natural filtration is studied by the methods of minimal dimension of image spaces and the derivation superalgebras. In view of the above invariance of the natural filtrations we describe the intrinsic properties of these modular Lie superalgebras.

This paper is arranged as follows. A brief summary of the relevant concepts and notations in finite-dimensional modular Lie superalgebras $W(n, m)$ and $H(n, m)$ is presented in Section 2. In Section 3, by using the ad-nilpotent elements of the Lie superalgebras $W(n, m)$, we show that the natural filtration of $W(n, m)$ is invariant under their automorphisms. In Section 4, the intrinsic properties with respect to the natural filtration of finite-dimensional modular Lie superalgebras $H(n, m)$ are investigated. Besides, the isomorphic relation between $H(n, m)$ and $H\left(n^{\prime}, m^{\prime}\right)$ is also proved by the method of the natural filtration.

\section{Preliminaries}

Throughout this paper, $\mathbb{F}$ denotes an algebraic closed field of characteristic $p>2$, $n$ is an integer greater than 1 . Let $\mathbb{Z}, \mathbb{N}$ and $\mathbb{N}_{0}$ denote the sets of integers, positive integers and nonnegative integers. Let $\mathbb{Z}_{2}=\{\overline{0}, \overline{1}\}$ be the residue class ring of integers modulo 2 .

Let $\Lambda(n)$ be the Grassmann algebra over $\mathbb{F}$ in $n$ variables $x_{1}, x_{2}, \ldots, x_{n}$. Set $\mathbb{B}_{k}=\left\{\left\langle i_{1}, i_{2}, \ldots, i_{k}\right\rangle \mid 1 \leq i_{1}<i_{2}<\cdots<i_{k} \leq n\right\}$ and $\mathbb{B}(n)=\bigcup_{k=0}^{n} B_{k}$, where $B_{0}=\varnothing$. For $u=\left\langle i_{1}, i_{2}, \ldots, i_{k}\right\rangle \in \mathbb{B}(k)$, set $|u|=k,\{u\}=\left\{i_{1}, i_{2}, \ldots, i_{k}\right\}$, and $x^{u}=x_{i_{1}} x_{i_{2}} \cdots x_{i_{k}}\left(|\varnothing|=0, x^{\varnothing}=1\right)$. Then $\left\{x^{u} \mid u \in \mathbb{B}(n)\right\}$ is an $\mathbb{F}$-basis of $\Lambda(n)$.

Let $\Pi$ denote the prime field of $\mathbb{F}$, that is, $\Pi=\{0,1, \ldots, p-1\}$. Suppose that $\left\{z_{1}, z_{2}, \ldots, z_{m}\right\}$ is a $\Pi$-linearly independent finite subset of $\mathbb{F}$. Let $G=$ 
$\left\{\sum_{i=1}^{m} \lambda_{i} z_{i} \mid \lambda_{i} \in \Pi\right\}$. Then $G$ is an additive subgroup of $\mathbb{F}$. Let $\mathbb{F}\left[y_{1}, y_{2}, \ldots, y_{m}\right]$ be the truncated polynomial algebra satisfying $y_{i}^{p}=1$ for all $i=1,2, \ldots, m$. For every element $\lambda=\sum_{i=1}^{m} \lambda_{i} z_{i} \in G$, define $y^{\lambda}=y_{1}^{\lambda_{1}} y_{2}^{\lambda_{2}} \cdots y_{m}^{\lambda_{m}}$. Then $y^{\lambda} y^{\eta}=y^{\lambda+\eta}$ for all $\lambda, \eta \in G$. Let $\mathbb{T}(m)$ denote $\mathbb{E}\left[y_{1}, y_{2}, \ldots, y_{m}\right]$. Then $\mathbb{T}(m)=\left\{\sum_{\lambda \in G} a_{\lambda} y^{\lambda} \mid a_{\lambda} \in \mathbb{F}\right\}$. We denote the tensor product by $U=\Lambda(n) \otimes \mathbb{T}(m)$. Then $U$ is an associative superalgebra with $\mathbb{Z}_{2}$-gradation induced by the trivial $\mathbb{Z}_{2}$-gradation of $\mathbb{T}(m)$ and the natural $\mathbb{Z}_{2}$-gradation of $\Lambda(n)$, that is, $\mathcal{U}_{=} \boldsymbol{U}_{\overline{0}} \oplus \mathcal{U}_{\overline{1}}$, where $\boldsymbol{U}_{\overline{0}}=\Lambda(n)_{\overline{0}} \otimes \mathbb{T}(m)$ and $\mho_{\overline{1}}=\Lambda(n)_{\overline{1}} \otimes \mathbb{T}(m)$.

For $f \in \Lambda(n)$ and $\alpha \in \mathbb{T}(m)$, we abbreviate $f \otimes \alpha$ as $f \alpha$. Then the elements $x^{u} y^{\lambda}$ with $u \in \mathbb{B}(n)$ and $\lambda \in G$ form an $\mathbb{F}$-basis of $U$. It is easy to see that $U=\bigoplus_{i=0}^{n} \varkappa_{i}$ is a $\mathbb{Z}$-graded superalgebra, where $U_{i}=\operatorname{span}_{\mathbb{F}}\left\{x^{u} y^{\lambda}|u \in \mathbb{B}(n)| u \mid,=i, \lambda \in G\right\}$. In particular, $\mathcal{U}_{0}=\mathbb{T}(m)$ and $\boldsymbol{U}_{n}=\operatorname{span}_{\mathbb{F}}\left\{x^{\pi} y^{\lambda} \mid \lambda \in G\right\}$, where $\pi:=\langle 1,2, \ldots, n\rangle \in \mathbb{B}(n)$.

In this paper, if $A=A_{\overline{0}} \oplus A_{\overline{1}}$ is a superalgebra (or $\mathbb{Z}_{2}$-graded linear space), let $\operatorname{hg}(A)=A_{\overline{0}} \cup A_{\overline{1}}$; that is, $\operatorname{hg}(A)$ is the set of all $\mathbb{Z}_{2}$-homogeneous elements of $A$. If $\operatorname{deg} x$ occurs in some expression, we regard $x$ as a $\mathbb{Z}_{2}$-homogeneous element and $\operatorname{deg} x$ as the $\mathbb{Z}_{2}$-degree of $x$. Let $A=\bigoplus_{i=-r}^{n} A_{i}$ be a $\mathbb{Z}$-graded superalgebra. If $x \in A_{i}$, we call $x$ a $\mathbb{Z}$-homogeneous element, $i$ the $\mathbb{Z}$-degree of $x$ and $\operatorname{set} \operatorname{zd}(x)=i$. If $y \in A$, let $\mu(y)$ denote the nonzero $\mathbb{Z}$-homogeneous part of $y$ with the least $\mathbb{Z}$-degree.

Let $\operatorname{pl}(A)=\operatorname{pl}_{\overline{0}}(A) \oplus \operatorname{pl}_{\overline{1}}(A)$ denote the general linear Lie superalgebra of the $\mathbb{Z}_{2}$-graded space $A$. For $\varphi \in \operatorname{pl}_{\theta}(A)$ with $\theta \in \mathbb{Z}_{2}$, if

$$
\varphi(x y)=\varphi(x) y+(-1)^{\theta \operatorname{deg} x} x \varphi(y)
$$

for all $x \in \operatorname{hg}(A)$ and $y \in A$, then $\varphi$ is called a derivation of $A$ with $\mathbb{Z}_{2}$-degree $\theta$. Let $\operatorname{Der}_{\theta} A$ denote the set of all derivations of $A$ with $\mathbb{Z}_{2}$-degree $\theta$. Then $\operatorname{Der} A=$ $\operatorname{Der}_{0}^{-} A \oplus \operatorname{Der}_{1}^{-} A$ is a subalgebra of $\operatorname{pl}(A)$ (see [Scheunert 1979]), which is called the derivation superalgebra of $A$.

Set $\mathrm{Y}=\{1,2, \ldots, n\}$. Given $i \in \mathrm{Y}$, let $\partial / \partial x_{i}$ be the partial derivative on $\Lambda(n)$ with respect to $x_{i}$. For $i \in Y$, let $D_{i}$ be the linear transformation on $U$ such that $D_{i}\left(x^{u} y^{\lambda}\right)=\left(\partial x^{u} / \partial x_{i}\right) y^{\lambda}$ for all $u \in \mathbb{B}(n)$ and $\lambda \in G$. Then $D_{i} \in \operatorname{Der}_{1}^{-} \mathcal{u}$ for all $i \in \mathrm{Y}$ since $\partial / \partial x_{i} \in \operatorname{Der}_{1}^{-}(\Lambda(n))$.

Suppose that $u \in \mathbb{B}_{k} \subseteq \mathbb{B}(n)$ and $i \in \mathrm{Y}$. When $i \in\{u\}$, we denote the uniquely determined element of $\mathbb{B}_{k-1}$ satisfying $\{u-\langle i\rangle\}=\{u\} \backslash\{i\}$ by $u-\langle i\rangle$, and denote the number of integers less than $i$ in $\{u\}$ by $\tau(u, i)$. When $i \notin\{u\}$, we set $\tau(u, i)=0$ and $x^{u-\langle i\rangle}=0$. Therefore, $D_{i}\left(x^{u}\right)=(-1)^{\tau(u, i)} x^{u-\langle i\rangle}$ for any $i \in \mathrm{Y}$ and $u \in \mathbb{B}(n)$.

We define $(f D)(g)=f D(g)$ for $f, g \in \operatorname{hg}(U)$ and $D \in \operatorname{hg}(\operatorname{Der} U)$. Since the multiplication of $U$ is supercommutative, it follows that $f D$ is a derivation of $U$. Let

$$
W(n, m)=\operatorname{span}_{\mathbb{F}}\left\{x^{u} y^{\lambda} D_{i} \mid u \in \mathbb{B}(n), \lambda \in G, i \in \mathrm{Y}\right\} .
$$


Then $W(n, m)$ is a finite-dimensional Lie superalgebra contained in Der $u$. A direct computation shows that

$$
\left[f D_{i}, g D_{j}\right]=f D_{i}(g) D_{j}-(-1)^{\operatorname{deg} f D_{i} \operatorname{deg} g D_{j}} g D_{j}(f) D_{i},
$$

where $f, g \in \operatorname{hg}(U)$ and $i, j \in \mathrm{Y}$.

Let $D_{H}: U \rightarrow W(n, m)$ be the linear mapping such that for every $f \in \operatorname{hg}(u)$, $D_{H}(f)=\sum_{i=1}^{n} f_{i} D_{i}$, where $f_{i}=(-1)^{\operatorname{deg} f} D_{i}(f)$. It is easy to see that $D_{H}$ is an even linear mapping and $D_{i}\left(f_{j}\right)=-D_{j}\left(f_{i}\right)$ for all $i, j \in \mathrm{Y}$. Let $\bar{H}(n, m)=$ $\left\{D_{H}(f) \mid f \in \mathcal{U}\right\}$ and $H(n, m)=\left\{D_{H}(f) \mid f \in \bigoplus_{i=0}^{n-1} U_{i} i\right\}$. Then $H(n, m)$ is a finite-dimensional Hamiltonian Lie superalgebra, with a $\mathbb{Z}$-gradation $H(n, m)=$ $\bigoplus_{i=-1}^{n-3} H_{i}(n, m)$, where $H_{i}(n, m)=\left\{D_{H}\left(x^{u} y^{\lambda}\right)|u \in \mathbb{B}(n)| u \mid,=i+2, \lambda \in G\right\}$. It was shown in [Ren et al. 2011] that $H(n, m)$ is a subalgebra of $W(n, m)$ and that

$$
\begin{aligned}
{\left[D_{H}(f), D_{H}(g)\right] } & =D_{H}\left(\sum_{i=1}^{n}(-1)^{\operatorname{deg} f} D_{i}(f) D_{i}(g)\right), \\
{\left[D_{j}, D_{H}(f)\right] } & =D_{H}\left(D_{j}(f)\right),
\end{aligned}
$$

where $f, g \in \operatorname{hg}(U)$ and $j \in Y$.

Let $\Theta:=T(m)^{m}=T(m) \times \cdots \times T(m)$. For every $\theta=\left(h_{1}(y), \ldots, h_{m}(y)\right) \in \Theta$, we define $\tilde{\theta}: G \rightarrow T(m)$ by $\tilde{\theta}(\lambda)=\sum_{j=1}^{m} \lambda_{j} h_{j}(y)$ for $\lambda=\sum_{j=1}^{m} \lambda_{j} z_{j} \in G$. It is easy to check that $\tilde{\theta}(\lambda+\eta)=\tilde{\theta}(\lambda)+\tilde{\theta}(\eta)$ for $\lambda, \eta \in G$. For every $\theta \in \Theta$, let $D_{\theta}: H(n, m) \rightarrow H(n, m)$ be the linear mapping given by $D_{\theta} D_{H}\left(x^{u} y^{\lambda}\right)=$ $\tilde{\theta}(\lambda) D_{H}\left(x^{u} y^{\lambda}\right)$ for $x^{u} y^{\lambda} \in \mathcal{U}$. A direct verification shows that $D_{\theta} \in \operatorname{Der}_{0}^{-} H$ for all $\theta \in \Theta$. Put $\Omega:=\left\{D_{\theta} \mid \theta \in \Theta\right\}$.

\section{The natural filtration of $W(n, m)$}

In this section, $W$ always denotes Lie superalgebras $W(n, m)$. Then $W=\bigoplus_{k=-1}^{n-1} W_{k}$ is $\mathbb{Z}$-graded, where $W_{k}=\operatorname{span}_{\mathbb{F}}\left\{x^{u} y^{\lambda} D_{j}|| u \mid=k+1, j \in \mathrm{Y}\right\}$.

Adopting the notion of [Jin 1992], the element $x$ of Lie superalgebra $L$ is called ad-nilpotent if ad $x$ is a nilpotent linear transformation. The set of all ad-nilpotent elements of $L$ is denoted by $\operatorname{nil}(L)$. Let $L_{(j)}=\bigoplus_{k \geq j} L_{k}$; then $\left\{L_{(j)} \mid j \geq-1\right\}$ is the natural filtration of $L$. If $L$ is $\mathbb{Z}$-graded and finite-dimensional, then $L_{-1} \subseteq \operatorname{nil}(L)$ and $L_{(1)} \subseteq \operatorname{nil}(L)$.

Let $M_{n}(\mathbb{F})$ denote the set of all $n \times n$ matrices over $\mathbb{F}$. Notice that $\operatorname{dim} T(m)=p^{m}$. Without loss of generality, we may suppose that $\left\{y_{1}, \ldots, y_{p^{m}}\right\}$ is a standard $\mathbb{F}$-basis of $T(m)$. If

$$
z=\sum_{i, j=1}^{n} \sum_{q=1}^{p^{m}} a_{i j q} x_{i} y_{q} D_{j} \in W_{0},
$$

where $a_{i j q} \in \mathbb{F}$, let 


$$
\rho(z)=\left(\begin{array}{ccc}
A_{1} & & \\
& \ddots & \\
& & A_{p^{m}}
\end{array}\right)_{n p^{m} \times n p^{m}}
$$

where $A_{q}=\left(a_{i j q}\right)_{n \times n} \in M_{n}(\mathbb{F})$.

Lemma 3.1. Suppose that $z=\sum_{i, j=1}^{n} \sum_{q=1}^{p^{m}} a_{i j q} x_{i} y_{q} D_{j} \in W_{0}$. If $z$ is ad-nilpotent, then $\rho(z)$ is a nilpotent matrix.

Proof. Let $\Gamma$ be the representation of $W_{0}$ with values in $W_{-1}$. Then $\Gamma(z)=\operatorname{ad} z$ and the matrix of $\Gamma(z)$ over the basis $\left\{y_{1} D_{1}, \ldots, y_{1} D_{n}, \ldots, y_{p^{m}} D_{1}, \ldots, y_{p^{m}} D_{n}\right\}$ of $W_{-1}$ is

$$
A=\left(\begin{array}{ccc}
-\left(A_{1}\right)^{t} & & \\
& \ddots & \\
& & -\left(A_{p^{m}}\right)^{t}
\end{array}\right)_{n p^{m} \times n p^{m}}
$$

where $A_{q}=\left(a_{i j q}\right)_{n \times n} \in M_{n}(\mathbb{F})$. Since $z$ is ad-nilpotent, the representation $\Gamma(z)$ is a nilpotent linear transformation. This implies that $A$ is nilpotent. Therefore, $\rho(z)=-A^{t}$ is a nilpotent matrix.

Lemma 3.2. Let $z=\sum_{i=k}^{n-1} z_{i}$, where $z_{i} \in W_{i}$ and $k \leq n-1$. If $z \in \operatorname{nil}(W)$ and $k \geq 0$, then $z_{k} \in \operatorname{nil}(W)$.

Proof. Suppose that $z=z_{k}+z^{\prime}$, where $z_{k} \in W_{k}$ and $z^{\prime} \in \bigoplus_{i=k+1}^{n-1} W_{i} \subseteq W_{(k+1)}$. Since $z \in \operatorname{nil}(W)$, we may assume that $(\operatorname{ad} z)^{t}=0$. Let $x$ is a $\mathbb{Z}$-homogeneous element of $W$ with $\mathbb{Z}$-degree $i$. Then $(\operatorname{ad} z)^{t}(x)=0$. On the other hand,

$$
(\operatorname{ad} z)^{t}(x)=\left(\operatorname{ad}\left(z_{k}+z^{\prime}\right)\right)^{t}(x)=\left(\operatorname{ad} z_{k}\right)^{t}(x)+h
$$

which implies $\left(\operatorname{ad} z_{k}\right)^{t}(x)+h=0$. It is easy to see that $\left(\operatorname{ad} z_{k}\right)^{t}(x) \in W_{(k t+i)}$ and $h \in W_{(k t+i+1)}=\bigoplus_{j \geq k t+i+1} W_{j}$. Thus $\left(\operatorname{ad} z_{k}\right)^{t}(x)=0$. Since $x$ is an arbitrary $\mathbb{Z}$-homogeneous element of $W$, we have $\left(\operatorname{ad} z_{k}\right)^{t}(W)=0$. Then $\left(\operatorname{ad} z_{k}\right)^{t}=0$, that is, $z_{k} \in \operatorname{nil}(W)$.

Suppose that $E_{i j}$ denotes the $n \times n$ matrix whose $(i, j)$ element is 1 and otherwise are zero. Obviously,

$$
E_{i j} E_{k l}=\delta_{j k} E_{i l}
$$

where $\delta_{j k}$ is the Kronecker delta.

If $z=\sum_{i, j=1}^{n} \sum_{q=1}^{p^{m}} a_{i j q} x_{i} y_{q} D_{j} \in W_{0}$, where $a_{i j q} \in \mathbb{F}$, then

$$
\rho(z)=\sum_{i, j=1}^{n} a_{i j 1} E_{i j}+\sum_{i, j=n+1}^{2 n} a_{i j 2} E_{i j}+\cdots+\sum_{i, j=n\left(p^{m}-1\right)+1}^{n p^{m}} a_{i j p^{m}} E_{i j} .
$$

Let $\Delta=\{z \in \operatorname{nil}(W) \mid \operatorname{ad} z(W) \subseteq \operatorname{nil}(W)\}$. 
Lemma 3.3. Suppose that $z=\sum_{i=-1}^{n-1} z_{i}$, where $z_{i} \in W_{i}$. If $z \in \Delta$, then $z_{-1}=z_{0}=0$. Proof. Suppose that $0 \neq z_{-1}=\sum_{i=1}^{n} \sum_{q=1}^{p^{m}} a_{i q} y_{q} D_{i}$, where $a_{i q} \in \mathbb{F}$. Let $a_{j q} \neq 0$ and $j, l \in \mathrm{Y}$ such that $j, l$ are distinct. We may assume that $d=\left[z_{-1}, x_{l} x_{j} D_{l}\right]$. A direct calculation shows that

$$
d=\left[\sum_{i=1}^{n} \sum_{q=1}^{p^{m}} a_{i q} y_{q} D_{i}, x_{l} x_{j} D_{l}\right]=\sum_{q=1}^{p^{m}}\left(a_{l q} x_{j} y_{q} D_{l}-a_{j q} x_{l} y_{q} D_{l}\right) .
$$

By (3-1) and (3-2), we have

$$
\begin{aligned}
(\rho(d))^{t}= & (-1)^{t}\left(a_{j 1}\right)^{t} E_{l l}+(-1)^{t-1} a_{l 1}\left(a_{j 1}\right)^{t-1} E_{j l} \\
& +(-1)^{t}\left(a_{(j+n) 2}\right)^{t} E_{(l+n)(l+n)}+(-1)^{t-1} a_{(l+n) 2}\left(a_{(j+n) 1}\right)^{t-1} E_{(j+n)(l+n)} \\
& +\cdots \\
& +(-1)^{t}\left(a_{\left.\left(j+p^{m}-n\right) p^{m}\right)^{t} E_{\left(l+p^{m}-n\right)\left(l+p^{m}-n\right)}}\right. \\
& +(-1)^{t-1} a_{\left(l+p^{m}-n\right) p^{m}}\left(a_{\left(j+p^{m}-n\right) p^{m}}\right)^{t-1} E_{\left(j+p^{m}-n\right)\left(l+p^{m}-n\right)} .
\end{aligned}
$$

Since $\left(a_{j 1}\right)^{t} \neq 0$, we have $(\rho(d))^{t} \neq 0$. So $\rho(d)$ is not a nilpotent matrix. By Lemma 3.1, it shows that $d \notin \operatorname{nil}(W)$. By Lemma 3.2, we have $\left[z, x_{l} x_{j} D_{l}\right] \notin \operatorname{nil}(W)$. Then $z \notin \Delta$. This contradicts $z \in \Delta$, and proves our assertion that $z_{-1}=0$.

Assume that $z_{0} \neq 0$. Let $z_{0}=\sum_{i, j=1}^{n} \sum_{q=1}^{p^{m}} a_{i j q} x_{i} y_{q} D_{j}, a_{i j q} \in \mathbb{F}$ and

$$
\begin{aligned}
& l=\min \left\{i \mid a_{i j \lambda} \neq 0, i, j \in \mathrm{Y}\right\}, \\
& t=\min \left\{j \mid a_{i j \lambda} \neq 0, i, j \in \mathrm{Y}\right\} .
\end{aligned}
$$

(i) Suppose that $l \leq t$. Let

$$
k=\max \left\{j \mid a_{l j \lambda} \neq 0, j \in \mathrm{Y}\right\} .
$$

Then $a_{l k q} \neq 0$. It is easy to see that $t \leq k$. Since $l \leq t$, we have $l \leq k$. Therefore,

$$
z_{0}=\sum_{j=t}^{k} \sum_{q=1}^{p^{m}} a_{l j q} x_{l} y_{q} D_{j}+\sum_{i=l+1}^{n} \sum_{j=t}^{n} \sum_{q=1}^{p^{m}} a_{i j q} x_{i} y_{q} D_{j} .
$$

Assume that $l=k$. It follows from $t \leq k$ that $t \leq l$. Then $t=l$, which implies that

$$
z_{0}=\sum_{q=1}^{p^{m}} a_{l l q} x_{l} y_{q} D_{l}+\sum_{i=l+1}^{n} \sum_{j=t}^{n} \sum_{q=1}^{p^{m}} a_{i j q} x_{i} y_{q} D_{j}
$$


Therefore,

$$
\begin{aligned}
& \rho\left(z_{0}\right)=a_{l l 1} E_{l l}+\sum_{i=l+1}^{n} \sum_{j=t}^{n} a_{i j 1} E_{i j} \\
& +a_{(l+n)(l+n) 2} E_{(l+n)(l+n)}+\sum_{i=l+1+n}^{2 n} \sum_{j=t+n}^{2 n} a_{i j 2} E_{i j} \\
& +\cdots \\
& +a_{\left(l+n\left(p^{m}-1\right)\right)\left(l+n\left(p^{m}-1\right)\right) p^{m}} E_{(l+n)(l+n)} \\
& +\sum_{i=l+1+n\left(p^{m}-1\right)}^{n p^{m}} \sum_{j=t+n\left(p^{m}-1\right)}^{n p^{m}} a_{i j p^{m}} E_{i j} \\
& =\left(\begin{array}{lllll}
A_{1} & & & \\
B_{1} & C_{1} & & \\
& \ddots & & \\
& & A_{p^{m}} & \\
& & B_{p^{m}} & C_{p^{m}}
\end{array}\right)_{n p^{m} \times n p^{m}},
\end{aligned}
$$

where $A_{k}=a_{(l+(k-1) n)(l+(k-1) n) q} E_{(l+(k-1) n)(l+(k-1) n)}$ is an $(l+(k-1) n)$-by$(l+(k-1) n)$ matrix and $q \in\left\{1, \ldots, p^{m}\right\}$. Since $a_{l l 1} \neq 0$, we have $A_{1}$ is not a nilpotent matrix. Then $\rho\left(z_{0}\right)$ is not a nilpotent matrix and $z_{0} \notin \operatorname{nil}(W)$. Lemma 3.2 shows that $z \notin \operatorname{nil}(W)$. This is in contradiction with $z \in \Delta$; thus $l<k$.

Suppose that $h \in \mathrm{Y}$ and $h \neq l, k$. Let $d=\left[z_{0}, x_{k} D_{l}\right]$. From (2-1), we obtain

$$
d=\sum_{q=1}^{p^{m}}\left(a_{l k q} x_{l} y_{q} D_{l}+\sum_{i=l+1}^{n} a_{i k q} x_{i} y_{q} D_{l}-\sum_{j=t}^{k} a_{l j q} x_{k} y_{q} D_{j}\right) .
$$

Since $l<k, \rho(d)$ also has the form

$$
\left(\begin{array}{llll}
A_{1} & & & \\
B_{1} & C_{1} & & \\
& & \ddots & \\
& & A_{p^{m}} \\
& & B_{p^{m}} & C_{p^{m}}
\end{array}\right)_{n p^{m} \times n p^{m}} .
$$

It follows from $a_{l k q} \neq 0$ that $A_{1}$ is not a nilpotent matrix. Then $\rho(d)$ is not nilpotent. So $z \notin \operatorname{nil}(W)$ and $\left[z, x_{k} D_{l}\right] \notin \operatorname{nil}(W)$. This is in contradiction with $z \in \Delta$.

(ii) Suppose that $t<l$. Let $k=\max \left\{i \mid a_{i t \lambda} \neq 0\right\}$ and $d^{\prime}=\left[z, x_{t} D_{k}\right]$. Imitating (i), we may prove that $\rho\left(d^{\prime}\right)$ is also not nilpotent. The desired result follows.

Lemma 3.4. (i) If $z \in W_{0} \cap \operatorname{nil}(W)$ and $h \in W_{(1)}$, then $z+h \in \operatorname{nil}(W)$.

(ii) If $i, j$ are distinct elements of $Y$, then $x_{i} y^{\lambda} D_{j} \in \operatorname{nil}(W)$ for all $\lambda \in G$. 
(iii) If $i, j, k$ are distinct elements of $\mathrm{Y}$, then $a x_{j} y^{\lambda} D_{k}+b x_{i} y^{\eta} D_{k} \in \operatorname{nil}(W)$ and $x_{i} x_{j} y^{\lambda} D_{k} \in \Delta$, where $a, b \in \mathbb{F}$ and $\lambda, \eta$ are arbitrary elements of $G$.

Proof. (i) A direct verification shows that $\{\operatorname{ad} z\} \cup\left\{\operatorname{ad} W_{(1)}\right\}$ is a weakly closed subset of nilpotent elements of $\mathrm{pl}(W)$. It was shown in [Jacobson 1962, Theorem 1 of Chapter II] that each element of $\operatorname{span}_{\mathbb{E}}\left(\{\operatorname{ad} z\} \cup\left\{\operatorname{ad} W_{(1)}\right\}\right)$ is a nilpotent linear transformation of $W$. Then $\operatorname{ad} z+\operatorname{ad} h$ is nilpotent. So $z+h$ is ad-nilpotent.

(ii) To prove $\left(\operatorname{ad} x_{i} y^{\lambda} D_{j}\right)^{p}=0$, we may assume without loss of generality that $i<j$. Set $\eta$ is an arbitrary element of $G$. If $k \neq i$, then

$$
\begin{aligned}
\left(\operatorname{ad} x_{i} y^{\lambda} D_{j}\right)^{2}\left(x^{u} y^{\eta} D_{k}\right) & =\left[x_{i} y^{\lambda} D_{j},\left[x_{i} y^{\lambda} D_{j}, x^{u} y^{\eta} D_{k}\right]\right] \\
& =(-1)^{\tau(u, j)}\left[x_{i} y^{\lambda} D_{j}, x_{i} x^{u-\langle j\rangle} y^{\lambda+\eta} D_{k}\right] \\
& =0 .
\end{aligned}
$$

In the case of $k=i$, we have

$$
\begin{aligned}
\left(\operatorname{ad} x_{i} y^{\lambda} D_{j}\right)^{3}\left(x^{u} y^{\eta} D_{k}\right) & =\left[x_{i} y^{\lambda} D_{j},\left[x_{i} y^{\lambda} D_{j},\left[x_{i} y^{\lambda} D_{j}, x^{u} y^{\eta} D_{i}\right]\right]\right] \\
& =\left[x_{i} y^{\lambda} D_{j},\left[x_{i} y^{\lambda} D_{j},(-1)^{\tau(u, j)} x_{i} x^{u-\langle j\rangle} y^{\lambda} D_{i}-x^{u} y^{\lambda+\eta} D_{j}\right]\right] \\
& =(-1)^{\tau(u, j)}\left[x_{i} y^{\lambda} D_{j},-x_{i} x^{u-\langle j\rangle} y^{\lambda} D_{j}-x_{i} x^{u-\langle j\rangle} y^{2 \lambda+\eta} D_{j}\right] \\
& =0 .
\end{aligned}
$$

For $p>2$ we get $\left(\operatorname{ad} x_{i} y^{\lambda} D_{j}\right)^{p}\left(x^{u} y^{\eta} D_{k}\right)=0$. Therefore $\left(\operatorname{ad} x_{i} y^{\lambda} D_{j}\right)^{p}(W)=0$. This yields $\left(\operatorname{ad} x_{i} y^{\lambda} D_{j}\right)^{p}=0$. Thus $x_{i} y^{\lambda} D_{j} \in \operatorname{nil}(W)$.

(iii) According to (ii) and $\left[x_{j} y^{\lambda} D_{k}, x_{i} y^{\eta} D_{k}\right]=0,\left\{\operatorname{ad} x_{j} y^{\lambda} D_{k}, \operatorname{ad} x_{i} y^{\eta} D_{k}\right\}$ is a weakly closed subset of nilpotent elements of $\mathrm{pl}(\mathrm{W})$. So $a x_{j} y^{\lambda} D_{k}+b x_{i} y^{\eta} D_{k} \in$ $\operatorname{nil}(W)$, where $a, b \in \mathbb{F}$.

Suppose that $l \in \mathrm{Y} \backslash\{i, j, k\}$. Then $x_{i} x_{j} y^{\lambda} D_{k} \in W_{(1)} \subseteq \operatorname{nil}(W)$. Let $z=\sum_{i=-1}^{n-1} z_{i}$, where $z_{i} \in W_{i}$. Without loss, we may assume that $\left[x_{i} x_{j} y^{\lambda} D_{k}, z\right]=f_{0}+f_{1}$, where $f_{0}=\left[x_{i} x_{j} y^{\lambda} D_{k}, z_{-1}\right] \in W_{0}$ and $f_{1} \in W_{(1)}$. Let $z_{-1}=\sum_{l=1}^{n} \sum_{\eta \in G} a_{l \eta} y^{\eta} D_{l}$. Then

$$
f_{0}=\left[x_{i} x_{j} y^{\lambda} D_{k}, \sum_{l=1}^{n} \sum_{\eta \in G} a_{l \eta} y^{\eta} D_{l}\right]=-\sum_{\eta \in G}\left(a_{i \eta} x_{j} y^{\lambda+\eta} D_{k}+a_{j \eta} x_{i} y^{\lambda+\eta} D_{k}\right) \text {. }
$$

It follows that $f_{0} \in W_{0} \cap \operatorname{nil}(W)$. Statement (i) shows that $f_{0}+f_{1} \in \operatorname{nil}(W)$. We finally obtain $x_{i} x_{j} y^{\lambda} D_{k} \in \Delta$ for all $\lambda \in G$.

Let $Q=\{z \in \operatorname{nil}(W) \mid \operatorname{ad} z(\Delta) \subseteq \Delta\}$.

\section{Lemma 3.5. \\ $Q=W_{(1)}$.}

Proof. By the definition of $\Delta$, we have $W_{(2)} \subseteq \Delta$. Lemma 3.3 show that $\Delta \subseteq W_{(1)}$. Then $\left[W_{(1)}, \Delta\right] \subseteq\left[W_{(1)}, W_{(1)}\right] \subseteq W_{(2)} \subseteq \Delta$. Thus $W_{(1)} \subseteq Q$. 
Next we will prove $Q \subseteq W_{(1)}$. Let $z \in Q$ and $z=\sum_{i=-1}^{n-1} z_{i}$, where $z_{i} \in W_{i}$. Assume that $z_{-1}=\sum_{l=1}^{n} \sum_{\lambda \in G} a_{l \lambda} y^{\lambda} D_{l} \neq 0, a_{l \lambda} \in \mathbb{F}$. Without loss of generality, we may suppose that $a_{i} \neq 0$. Let $d=x_{i} x_{j} y^{\eta} D_{k}$, where $i, j, k$ are distinct elements of $\mathrm{Y}$ and $\eta$ is an arbitrary element of $G$. By Lemma 3.4 (iii), we have $d \in \Delta$. Let $[z, d]=h_{0}+h_{1}$, where $h_{0}=\left[z_{-1}, d\right] \in W_{0}$ and $h_{1} \in W_{(1)}$. Since $a_{i} \neq 0$, we have $h_{0}=\sum_{\lambda \in G}\left(a_{i \lambda} x_{j} y^{\lambda+\eta} D_{k}-a_{j \lambda} x_{i} y^{\lambda+\eta} D_{k}\right) \neq 0$. Lemma 3.3 implies that $h_{0}+h_{1} \notin \Delta$. It is a contradiction to $z \in Q$. Hence $z_{-1}=0$.

Assume that $0 \neq z_{0}=\sum_{i, j=1}^{n} \sum_{q=1}^{p^{m}} a_{i j \lambda} x_{i} y_{q} D_{j}, a_{i j q} \in \mathbb{F}$ and suppose that $l$ and $t$ are as the definitions in (3-3) and (3-4). We may suppose that $l \leq t$ (the proof is similar to the case $t<l$ ) and let $k$ be as definition in (3-5). Similar to the first part of the proof in Lemma 3.3, we have $l<k$. Suppose that $h \in \mathrm{Y} \backslash\{l, k, t\}$ and $d_{1}=x_{k} x_{h} D_{l}$. Lemma 3.4 (iii) shows that $d_{1} \in \Delta$. Let $\left[z, d_{1}\right]=g_{1}+g_{2}$, where $g_{1}=\left[z_{0}, d_{1}\right] \in W_{1}$ and $g_{2} \in W_{(2)}$. Using (2-1), we have

$$
g_{1}=\sum_{q=1}^{p^{m}}\left(a_{l k q} x_{l} x_{h} y_{q} D_{l}-\sum_{i=l+1}^{n} a_{i h q} x_{i} x_{k} y_{q} D_{l}-\sum_{j=t}^{k} a_{l j q} x_{k} x_{h} y_{q} D_{j}\right) \text {. }
$$

If $h<t$, then $a_{i h q}=0$ in the above equality, where $i \in \mathrm{Y} \backslash\{1, \ldots, l-1\}$. Thus

$$
\left[D_{h}, g_{1}\right]=-\sum_{q=1}^{p^{m}}\left(a_{l k q} x_{l} y_{q} D_{l}+\sum_{i=l+1}^{n} a_{i h q} x_{i} y_{q} D_{l}+a_{h h q} x_{k} y_{q} D_{l}-a_{l j q} x_{k} y_{q} D_{j}\right) \text {. }
$$

By (3-2), the matrix $\rho\left(\left[D_{h}, g_{1}\right]\right)$ has the form

$$
\left(\begin{array}{ccccc}
A_{1} & & & \\
B_{1} & C_{1} & & \\
& & \ddots & \\
& & A_{p^{m}} & \\
& & B_{p^{m}} & C_{p^{m}}
\end{array}\right)_{n p^{m} \times n p^{m}}
$$

as in Lemma 3.3. Since $a_{l k q} \neq 0, A_{1}$ is not a nilpotent matrix. This implies that $\rho\left(\left[D_{h}, g_{1}\right]\right)$ is not nilpotent. Hence $\left[D_{h}, g_{1}\right] \notin \operatorname{nil}(W)$. Lemma 3.2 shows that $\left[D_{h}, g_{1}+g_{2}\right] \notin \operatorname{nil}(W)$, that is, $\left[D_{h}, g_{1}+g_{2}\right] \notin \Delta$. It is contradict with $z \in Q$. Thus $z_{0}=0$. Therefore, $z \in W_{(1)}$ and $Q \subseteq W_{(1)}$.

It is easy to verify that $\Delta$ and $Q$ are invariant subspaces under the automorphisms of $W$. According to Lemma 3.5, $W_{(1)}$ is also invariant under the automorphisms of $W$. Since

$$
\begin{aligned}
& W_{(0)}=\left\{x \in W \mid\left[x, W_{(1)}\right] \subseteq W_{(1)}\right\}, \\
& W_{(i)}=\left\{x \in W_{i-1} \mid[x, W] \subseteq W_{(i-1)}\right\}, \quad i \geq 1,
\end{aligned}
$$

we easily obtain the following theorem.

Theorem 3.6. The natural filtration of $W$ is invariant under automorphisms of $W$. 
Let $\mathfrak{W}_{i}=W_{(i)} / W_{(i+1)}$ for $-1 \leq i \leq n-1$. Then $\mathfrak{W}_{i}$ is a $\mathbb{Z}$-graded space. Suppose that $\mathfrak{W}:=\bigoplus_{i=-1}^{n-1} \mathfrak{W}_{i}$; then $\mathfrak{W}$ is also a $\mathbb{Z}$-graded space. Let $x+W_{(i+1)} \in \mathfrak{W}_{i}$ and $y+W_{(j+1)} \in \mathfrak{W J}_{j}$. Define

$$
\left[x+W_{(i+1)}, y+W_{(j+1)}\right]:=[x, y]+W_{(i+j+1)} .
$$

It follows from $\left[\mathfrak{W}_{i}, \mathfrak{W}_{j}\right] \subseteq \mathfrak{W}_{i+j}$ that the operator $[$,$] on \mathfrak{W}$ is well-defined. There exists a linear expansion such that $\mathfrak{W}$ has a operator $[$,$] . A direct verification$ shows that $\mathfrak{W}$ is a Lie superalgebra with respect to the operator $[$,$] . The Lie$ superalgebras $\mathfrak{W}$ is called a Lie superalgebra induced by the natural filtration of $W$.

Lemma 3.7.

$$
\mathfrak{W} \cong W .
$$

Proof. Let $\phi: W \rightarrow \mathfrak{W}$ is a linear map such that $\phi(x)=x+W_{(i+1)}$, where $x \in W_{(i)} \backslash W_{(i+1)}$. A direct verification shows that $\phi$ is a homomorphism of Lie superalgebras. Suppose that $y \in \operatorname{ker} \phi$. If $y \neq 0$, then there exists $i \geq-1$ such that $y \in W_{(i)} \backslash W_{(i+1)}$. Since $\phi(y)=0$, we have $y+W_{(i+1)}=0$. Hence $y \in W_{(i+1)}$. That shows that $y=0$. Thus, $\operatorname{ker} \phi=0$. Therefore, $\phi$ is a monomorphism. It follows from $W$ is finite-dimensional that $\phi$ is an isomorphism.

The definition of $\phi$ shows that, for $i \geq-1$

$$
\begin{aligned}
\phi\left(W_{i}\right) & =\left\{x+W_{(i+1)} \mid x \in W_{i}\right\}=\left\{x+W_{(i+1)} \mid x \in W_{(i)}\right\} \\
& =W_{(i)} / W_{(i+1)}=\mathfrak{W}_{i} .
\end{aligned}
$$

Suppose that $m, n, m^{\prime}, n^{\prime}$ are elements of $\mathbb{N}$ greater than 1 . Similar to $W$, the Lie superalgebra $W\left(n^{\prime}, m^{\prime}\right)$ will be simply denoted by $W^{\prime}$. According to the definitions of $\Delta, Q$, and $\mathfrak{W}$ in $W$, we define $\Delta^{\prime}, Q^{\prime}$, and $\mathfrak{W}^{\prime}$ in $W^{\prime}$ using the same method.

Proposition 3.8. Suppose that $W \cong W^{\prime}$ and $\sigma$ is an isomorphism from $W$ to $W^{\prime}$. Then $\sigma\left(W_{(i)}\right)=W_{(i)}^{\prime}$ for all $i \geq-1$.

Proof. It is clear that $\sigma\left(W_{(-1)}\right)=W_{(-1)}^{\prime}$ and $\sigma(\operatorname{nil}(W))=\operatorname{nil}\left(W^{\prime}\right)$. A direct verification shows that $\sigma(\Delta)=\Delta^{\prime}$. Hence $\sigma(Q)=Q^{\prime}$. By virtue of Lemma 3.5, we have $Q=W_{(1)}$ and $Q^{\prime}=W_{(1)}^{\prime}$. Thus $\sigma\left(W_{(1)}\right)=W_{(1)}^{\prime}$. By (3-6) and (3-7), the desired result $\sigma\left(W_{(i)}\right)=W_{(i)}^{\prime}$ for all $i \geq-1$ is obtained.

Lemma 3.9. Suppose that $W \cong W^{\prime}$ and $\sigma$ is an isomorphism from $W$ to $W^{\prime}$. Then $\sigma$ induces an isomorphism $\tilde{\sigma}$ from $\mathfrak{W}$ to $\mathfrak{W}^{\prime}$ such that $\tilde{\sigma}\left(\mathfrak{W}_{i}\right)=\mathfrak{W}_{i}^{\prime}$ for all $i \geq-1$.

Proof. Define a linear map $\tilde{\sigma}: \mathfrak{W} \rightarrow \mathfrak{W}^{\prime}$ such that

$$
\tilde{\sigma}\left(x+W_{(i+1)}\right)=\sigma(x)+W_{(i+1)}^{\prime},
$$

where $x+W_{(i+1)} \in \mathfrak{W}_{i}$. Because of Proposition 3.8, the definition of $\tilde{\sigma}$ is reasonable 
and

$$
\begin{aligned}
\tilde{\sigma}\left(\left[x+W_{(i+1)}, y+W_{(j+1)}\right]\right) & =\sigma([x, y])+W_{(i+j+1)}^{\prime} \\
& =\left[\sigma(x)+W_{(i+1)}^{\prime}, \sigma(y)+W_{(j+1)}^{\prime}\right] \\
& =\left[\tilde{\sigma}\left(x+W_{(i+1)}^{\prime}\right), \tilde{\sigma}\left(y+W_{(j+1)}^{\prime}\right)\right] .
\end{aligned}
$$

Thus $\tilde{\sigma}$ is a homomorphism from $\mathfrak{W}$ to $\mathfrak{W}^{\prime}$. Clearly, $\tilde{\sigma}\left(\mathfrak{W}_{i}\right)=\mathfrak{W}_{i}^{\prime}$ for all $i \geq-1$. It shows that $\tilde{\sigma}$ is an epimorphism.

Suppose that $y \in \operatorname{ker} \tilde{\sigma}$; then $y \in \mathfrak{W}$. So we may suppose that $y=\sum_{i=-1}^{n-1} y_{i}$ and $y_{i} \in \mathfrak{W}_{i}$. Since $\mathfrak{W}_{i}=W_{(i)} / W_{(i+1)}$, let $y_{i}=z_{i}+W_{(i+1)}$, where $z_{i} \in W_{(i)}$. Hence $\tilde{\sigma}\left(y_{i}\right)=\sigma\left(z_{i}\right)+W_{(i+1)}^{\prime}$. It follows from $\tilde{\sigma}(y)=0$ that $\sum_{i=-1}^{n-1} \tilde{\sigma}\left(y_{i}\right)=0$. Thus $\tilde{\sigma}\left(y_{i}\right)=0$, that is, $\sigma\left(z_{i}\right)+W_{(i+1)}^{\prime}=0$. This shows $\sigma\left(z_{i}\right) \in W_{(i+1)}^{\prime}$. By Proposition 3.8, we have $z_{i} \in \sigma^{-1}\left(W_{(i+1)}^{\prime}\right)=W_{(i+1)}$. Then $y_{i}=z_{i}+W_{(i+1)}=0$ for $-1 \leq i \leq n-1$. Therefore, $y=0$ and $\operatorname{ker} \tilde{\sigma}=0$. Consequently, $\tilde{\sigma}$ is an isomorphism induced by $\sigma$ such that $\tilde{\sigma}\left(\mathfrak{W}_{i}\right)=\mathfrak{W}_{i}^{\prime}$ for all $i \geq-1$.

Theorem 3.10. $W \cong W^{\prime}$ if and only if $m=m^{\prime}$ and $n=n^{\prime}$.

Proof. Since the sufficiency is obvious, it suffices to prove the necessity. Suppose that $\phi: W \rightarrow \mathfrak{W}$ is the isomorphism given in the proof of Lemma 3.7. Similarly, there also exists the $\phi^{\prime}: W^{\prime} \rightarrow \mathfrak{W}^{\prime}$. According to (3-8) and Lemma 3.9, we have

$$
\phi\left(W_{i}\right)=\mathfrak{W}_{i}, \quad \phi^{\prime}\left(W_{i}^{\prime}\right)=\mathfrak{W}_{i}^{\prime}, \quad \tilde{\sigma}\left(\mathfrak{W}_{i}\right)=\mathfrak{W}_{i}^{\prime}
$$

for $-1 \leq i \leq n-1$. Let $\psi=\left(\phi^{\prime}\right)^{-1} \tilde{\sigma} \phi$. Then

$$
\psi\left(W_{i}\right)=\left(\phi^{\prime}\right)^{-1} \tilde{\sigma} \phi\left(W_{i}\right)=\left(\phi^{\prime}\right)^{-1} \tilde{\sigma}\left(\mathfrak{W}_{i}\right)=\left(\phi^{\prime}\right)^{-1}\left(\mathfrak{W}_{i}^{\prime}\right)=W_{i} .
$$

In particular, $\psi\left(W_{-1}\right)=W_{-1}^{\prime}$ and $\psi\left(W_{0}\right)=W_{0}^{\prime}$. Since $\operatorname{dim} W_{-1}=\operatorname{dim} W_{-1}^{\prime}$, we get $n p^{m}=n^{\prime} p^{m^{\prime}}$. By virtue of the definition of $W_{i}$, we have

$$
W_{0}=\operatorname{span}_{\mathbb{F}}\left\{x_{i} y^{\lambda} D_{j} \in W \mid i, j \in \mathrm{Y}, \lambda \in G\right\} .
$$

Thus $\operatorname{dim} W_{0}=n^{2} p^{m}$. By the same method used in $W_{0}$, we may obtain $\operatorname{dim} W_{0}^{\prime}=$ $n^{\prime 2} p^{m^{\prime}}$. According to $\operatorname{dim} W_{0}=\operatorname{dim} W_{0}^{\prime}$ and $n p^{m}=n^{\prime} p^{m^{\prime}}$, we have $n=n^{\prime}$ and $m=m^{\prime}$. In conclusion, the proof is completed.

\section{The natural filtration of $H(n, m)$}

In this section we will investigate the question of the natural filtration of the Lie superalgebras $H(n, m)$. For convenience, $H(n, m), \bar{H}(n, m)$ and $H_{i}(n, m)$ will be simply denoted by $H, \bar{H}$ and $H_{i}$.

Let $H_{(j)}=\bigoplus_{i \geq j} H_{i}$. Then

$$
H=H_{(-1)} \supseteq H_{(0)} \supseteq H_{(1)} \supseteq \cdots \supseteq H_{(n-3)} \supseteq H_{(n-2)}=0
$$


is a descending filtration of $H$, which is called the natural filtration of $H$. We also call $\left\{H_{(k)} \mid k \in \mathbb{Z}\right\}$ a filtration of $H$ for short, where $H_{(k)}=H$ if $k \leq-1$ and $H_{(k)}=0$ if $k \geq n-2$.

Lemma 4.1. Let $f_{i}=g_{i}+h_{i}$, where $f_{i}, g_{i}, h_{i} \in \mathcal{U}$ and $i=1, \ldots, k$. If the set $\left\{g_{i} \mid i=1, \ldots, k\right\}$ is linearly independent and

$$
\operatorname{span}_{\mathbb{F}}\left\{g_{i} \mid i=1, \ldots, k\right\} \cap \operatorname{span}_{\mathbb{F}}\left\{h_{i} \mid i=1, \ldots, k\right\}=0,
$$

then $\left\{f_{i} \mid i=1, \ldots, k\right\}$ is linearly independent.

Proof. If $\sum_{i=1}^{k} a_{i} f_{i}=0, a_{i} \in \mathbb{F}$, then $\sum_{i=1}^{k} a_{i} g_{i}=-\sum_{i=1}^{k} a_{i} h_{i}$. This shows that

$$
\sum_{i=1}^{k} a_{i} g_{i} \in \operatorname{span}_{\mathbb{F}}\left\{g_{i} \mid i=1, \ldots, k\right\} \cap \operatorname{span}_{\mathbb{F}}\left\{h_{i} \mid i=1, \ldots, k\right\}=0 .
$$

Since $\left\{g_{i} \mid i=1, \ldots, k\right\}$ is linearly independent, we obtain $a_{i}=0, i=1, \ldots, k$.

Lemma 4.2. If $h_{1}, h_{2}, \ldots, h_{k} \in H \backslash\{0\}$. If $\left\{h_{i} \mid i=1, \ldots, k\right\}$ is linearly dependent, then so is $\left\{\mu\left(h_{i}\right) \mid i=1, \ldots, k\right\}$.

Proof. Since $\left\{h_{i} \mid i=1, \ldots, k\right\}$ is linearly dependent, there exist $a_{1}, \ldots, a_{k} \in \mathbb{F}$ such that $\sum_{i=1}^{k} a_{i} h_{i}=0$ and some $a_{i}$ is not zero. We may suppose that $a_{1}, \ldots, a_{s} \neq 0$ and $a_{s+1}=\cdots=a_{k}=0$, where $1 \leq s \leq k$. Let

$$
\varepsilon=\min \left\{\operatorname{zd}\left(\mu\left(h_{i}\right)\right) \mid i=1, \ldots, s\right\} .
$$

Without loss of generality, we may suppose that $\operatorname{zd}\left(\mu\left(h_{i}\right)\right)=\varepsilon$ for $i=1, \ldots, t$ and $\operatorname{zd}\left(\mu\left(h_{i}\right)\right)>\varepsilon$ for $i=t+1, \ldots, s$. It follows from $\sum_{i=1}^{k} a_{i} h_{i}=0$ that $\sum_{i=1}^{k} a_{i} \mu\left(h_{i}\right)=0$. Since $a_{1}, \ldots, a_{t} \neq 0$, we obtain that $\left\{\mu\left(h_{i}\right) \mid i=1, \ldots, t\right\}$ is linearly dependent. Hence so is $\left\{\mu\left(h_{i}\right) \mid i=1, \ldots, k\right\}$.

Lemma 4.3. Let $g_{1}, g_{2}, \ldots, g_{k} \in \mathcal{U}$. If $\operatorname{zd}\left(\mu\left(g_{i}\right)\right) \geq 1, i=1, \ldots, k$, then $\left\{g_{i} \mid i=\right.$ $1, \ldots, k\}$ is linearly dependent if and only if $\left\{D_{H}\left(g_{i}\right) \mid i=1, \ldots, k\right\}$ is.

Proof. If $\left\{g_{i} \mid i=1, \ldots, k\right\}$ is linearly dependent, there exist $a_{1}, \ldots, a_{k} \in \mathbb{F}$, not all zero, such that $\sum_{i=1}^{k} a_{i} g_{i}=0$. Clearly, $D_{H}\left(\sum_{i=1}^{k} a_{i} g_{i}\right)=\sum_{i=1}^{k} a_{i} D_{H}\left(g_{i}\right)=0$. Hence $\left\{D_{H}\left(g_{i}\right) \mid i=1, \ldots, k\right\}$ is linearly dependent.

Conversely, we consider the sufficiency. Without loss of generality, we may suppose that $g=x^{u} y^{\lambda}$ for $u \in \mathbb{B}(n)$ and $\lambda \in G$ such that $D_{H}(g)=0$. Then

$$
D_{H}\left(x^{u} y^{\lambda}\right)=\sum_{i=1}^{n}(-1)^{|u|} D_{i}\left(x^{u}\right) y^{\lambda} D_{i} .
$$

Hence $D_{i}\left(x^{u}\right)=0$, which shows that $|u|=0$. Thus $\operatorname{ker}\left(D_{H}\right)=\mathbb{T}(m)$. Since the set $\left\{D_{H}\left(g_{i}\right) \mid i=1, \ldots, k\right\}$ is linearly dependent, there exist $a_{1}, \ldots, a_{k} \in \mathbb{F}$, 
not all zero, such that $\sum_{i=1}^{k} a_{i} D_{H}\left(g_{i}\right)=0$. Then $D_{H}\left(\sum_{i=1}^{k} a_{i} g_{i}\right)=0$. Hence $\sum_{i=1}^{k} a_{i} g_{i} \in \mathbb{T}(m)$. Notice that $\operatorname{zd}\left(\mu\left(g_{i}\right)\right) \geq 1, i=1, \ldots, k$; thus $\sum_{i=1}^{k} a_{i} g_{i}=0$, showing that $\left\{g_{i} \mid i=1, \ldots, k\right\}$ is linearly dependent.

For a superderivation $D$ of a Lie superalgebra $L$. Set $I(D)=\operatorname{dim}(\operatorname{Im}(D))$. If $T$ is a subset of superderivations of $L$, we define $I(T)=\min \{I(D) \mid 0 \neq D \in T\}$.

Theorem 4.4. Suppose that $T=\left.\operatorname{ad}(\operatorname{hg}(\bar{H}))\right|_{H}$, then $I(T) \geq n p^{m}$. Besides, $I\left(\operatorname{ad} D_{H}(g)\right)=n p^{m}$ if and only if $0 \neq D_{H}(g) \in \operatorname{span}_{\mathbb{F}}\left\{D_{H}\left(x^{\pi} y^{\lambda}\right) \mid \lambda \in G\right\}$, where $D_{H}(g) \in \operatorname{hg}(\bar{H})$.

Proof. For any $h \in \operatorname{hg}(\bar{H})$ we write ad $\left.h\right|_{H}$ simply as ad $h$. A direct calculation shows that

$$
\left[D_{H}\left(x^{\pi} y^{\lambda}\right), D_{H}\left(x^{v} y^{\eta}\right)\right]=D_{H}\left(\sum_{i=1}^{n}(-1)^{n} D_{i}\left(x^{\pi} y^{\lambda}\right) D_{i}\left(x^{v} y^{\eta}\right)\right)
$$

for $v \in \mathbb{B}(n)$ and $\lambda, \eta \in G$.

In the case of $|v| \geq 2$ we have

$$
D_{i}\left(x^{v} y^{\eta}\right)=(-1)^{\tau(v, i)} x^{v-\langle i\rangle} y^{\eta}, \quad D_{i}\left(x^{\pi} y^{\lambda}\right)=(-1)^{\tau(\pi, i)} x^{\pi-\langle i\rangle} y^{\lambda} .
$$

Clearly, $\{v-\langle i\rangle\} \in\{\pi-\langle i\rangle\}$. Then $\left[D_{H}\left(x^{\pi} y^{\lambda}\right), D_{H}\left(x^{v} y^{\eta}\right)\right]=0$ in this case.

In the case of $|v|=1$ we may suppose that $x^{v} y^{\eta}=x_{i} y^{\eta}$ for some $i \in \mathrm{Y}$. Then

$$
\begin{aligned}
{\left[D_{H}\left(x^{\pi} y^{\lambda}\right), D_{H}\left(x_{i} y^{\eta}\right)\right] } & =D_{H}\left(\sum_{j=1}^{n}(-1)^{n} D_{j}\left(x^{\pi} y^{\lambda}\right) D_{j}\left(x_{i} y^{\eta}\right)\right) \\
& =D_{H}\left((-1)^{n+\tau(\pi, i)} x^{\pi-\langle i\rangle} y^{\lambda+\eta}\right) .
\end{aligned}
$$

Since $\left\{x^{\pi-\langle i\rangle} y^{\lambda+\eta} \mid i \in \mathrm{Y}, \lambda, \eta \in G\right\}$ is a linearly independent set, Lemma 4.3 shows that $\left\{\left[D_{H}\left(x^{\pi} y^{\lambda}\right), D_{H}\left(x_{i} y^{\eta}\right)\right] \mid i \in \mathrm{Y}, \lambda, \eta \in G\right\}$ is linearly independent. Thus $I\left(\operatorname{ad} D_{H}(g)\right)=n p^{m}$.

Next we will consider the converse inclusion. Assume that $D_{H}\left(g_{0}\right) \in \operatorname{hg}(\bar{H})$ and $D_{H}\left(g_{0}\right) \notin \operatorname{span}_{\mathbb{F}}\left\{D_{H}\left(x^{\pi} y^{\lambda}\right) \mid \lambda \in G\right\}$. We want to prove that $I\left(\operatorname{ad} D_{H}\left(g_{0}\right)\right)>n p^{m}$. Suppose that $\mu\left(D_{H}\left(g_{0}\right)\right)=D_{H}(g)$. By Lemma 4.2, it suffices to prove that $I\left(\operatorname{ad} D_{H}(g)\right)>n p^{m}$.

Let $g=x^{u} y^{\lambda}$, where $u \in \mathbb{B}(n)$ and $\lambda \in G$. Then $1 \leq|u|<n$. There exist $v \in \mathbb{B}(n)$ and $\eta \in G$ such that $D_{H}\left(x^{v} y^{\eta}\right) \in H$. Then

$$
\left[D_{H}\left(x^{u} y^{\lambda}\right), D_{H}\left(x^{v} y^{\eta}\right)\right]=D_{H}\left(\sum_{i=1}^{n}(-1)^{|u|} D_{i}\left(x^{u} y^{\lambda}\right) D_{i}\left(x^{v} y^{\eta}\right)\right) .
$$

(1) Suppose that $|u|=1$ and $x^{u} y^{\lambda}=x_{i} y^{\lambda}$ for some $i \in \mathrm{Y}$, then

$$
\left[D_{H}\left(x_{i} y^{\lambda}\right), D_{H}\left(x^{v} y^{\eta}\right)\right]=-(-1)^{\tau(v, i)} D_{H}\left(x^{v-\langle i\rangle} y^{\lambda+\eta}\right) \text {. }
$$


Considering $x^{v-\langle i\rangle}$, the following statements hold:

$$
\left\{\begin{array}{cl}
D_{H}\left(x^{v-\langle i\rangle} y^{\lambda+\eta}\right)=0 & \text { if }|v|=0 \text { or } 1, \\
\operatorname{dim}\left\{x^{v-\langle i\rangle}\right\}=\mathrm{C}_{n-1}^{1} & \text { if }|v|=2, \\
\operatorname{dim}\left\{x^{v-\langle i\rangle}\right\}=\mathrm{C}_{n-1}^{2} & \text { if }|v|=3 \\
\vdots & \\
\operatorname{dim}\left\{x^{v-\langle i\rangle}\right\}=\mathrm{C}_{n-1}^{n-1} & \text { if }|v|=n .
\end{array}\right.
$$

Therefore,

$$
\operatorname{dim}\left\{x^{v-\langle i\rangle} y^{\lambda+\eta}\right\}=\left(\mathrm{C}_{n-1}^{1}+\mathrm{C}_{n-1}^{2}+\cdots+\mathrm{C}_{n-1}^{n-1}\right) p^{m}=\left(2^{n-1}-1\right) p^{m}>n p^{m} .
$$

(2) If $1<|u|<n$, then we suppose that $|u|=l$.

For $|v|=2$ we may suppose that $x^{v} y^{\eta}=x_{j} x_{k} y^{\eta}$, where $j, k$ are distinct elements of $\mathrm{Y}$ and $\eta$ is an arbitrary element of $G$. A direct calculation shows that $\left[D_{H}\left(x^{u} y^{\lambda}\right), D_{H}\left(x_{j} x_{k} y^{\eta}\right)\right]$ equals

$$
D_{H}\left((-1)^{|u|}\left((-1)^{\tau(u, j)} x^{u-\langle j\rangle} x_{k}-(-1)^{\tau(u, k)} x^{u-\langle k\rangle} x_{j}\right) y^{\lambda+\eta}\right) \text {. }
$$

Consider $\Upsilon=(-1)^{\tau(u, j)} x^{u-\langle j\rangle} x_{k}-(-1)^{\tau(u, k)} x^{u-\langle k\rangle} x_{j}$. Then the following statements hold:

$$
\begin{cases}\Upsilon=0 & \text { if } j, k \in u \text { or } j, k \notin u, \\ \Upsilon=(-1)^{\tau(u, j)} x^{(u \backslash\{j\}) \cup\{k\}} & \text { if } j \in u \text { and } k \notin u, \\ \Upsilon=(-1)^{\tau(u, k)} x^{(u \backslash\{k\}) \cup\{j\}} & \text { if } k \in u \text { and } j \notin u .\end{cases}
$$

Thus $\operatorname{dim} \Upsilon=l(n-l)$.

For $|v|=1$ we may suppose that $x^{v} y^{\eta}=x_{i} y^{\eta}$ for some $i \in \mathrm{Y}$. Then

$$
\left[D_{H}\left(x_{i} y^{\lambda}\right), D_{H}\left(x_{i} y^{\eta}\right)\right]=(-1)^{|u|+\tau(u, i)} D_{H}\left(x^{u-\langle i\rangle} y^{\lambda+\eta}\right), \quad i \in \mathrm{Y} .
$$

Hence $\operatorname{dim}\left(x^{u-\langle i\rangle}\right)=|u|=l$. It is clear to see that $l(n-l)+l>n$. Therefore, $I\left(\operatorname{ad} D_{H}(g)\right) \geq(l(n-l)+l) p^{m}>n p^{m}$.

Theorem 4.5. $I(\operatorname{hg}(\operatorname{Der} H))=n p^{m}$. Moreover, for $D \in \operatorname{hg}(\operatorname{Der}(H))$, we have $I(D)=n p^{m}$ if and only if $D$ is nonzero and lies in $\operatorname{span}_{\mathbb{F}}\left\{\operatorname{ad} D_{H}\left(x^{\pi} y^{\lambda}\right) \mid \lambda \in G\right\}$.

Proof. By virtue of Theorem 4.4, we have $I\left(\operatorname{ad} D_{H}\left(x^{\pi} y^{\lambda}\right)\right)=n p^{m}$. By [Ren et al. 2011, Proposition 3.7], we obtain

$$
\operatorname{Der} H=\operatorname{ad}\left(\bar{H}+\mathbb{F} y^{\lambda} h\right) \oplus \Omega,
$$

where $h=\sum_{i=1}^{n} x_{i} D_{i}$ and $\lambda \in G$. Hence $I(\operatorname{hg}(\operatorname{Der} H)) \leq n p^{m}$. Let $D \in \operatorname{hg}(\operatorname{Der} H)$ and $I(D) \leq n p^{m}$. Without loss of generality, we may suppose that

$$
D=\operatorname{ad} D_{H}(g)+a \operatorname{ad} y^{\lambda} h+\sum_{\theta \in \Theta} b_{\theta} D_{\theta},
$$


where $a, b_{\theta} \in \mathbb{F}$ and $D_{H}(g) \in \operatorname{hg}(\bar{H})$. Then

$$
\begin{aligned}
& D\left(D_{H}\left(x^{u} y^{\eta}\right)\right) \\
& \quad=\left[D_{H}(g), D_{H}\left(x^{u} y^{\eta}\right)\right]+a\left[\sum_{i=1}^{n} x_{i} y^{\lambda} D_{i}, D_{H}\left(x^{u} y^{\eta}\right)\right]+\sum_{\theta \in \Theta} b_{\theta} D_{\theta}\left(D_{H}\left(x^{u} y^{\eta}\right)\right)
\end{aligned}
$$

for all $u \in \mathbb{B}(n)$ and $\eta \in G$.

Next we will prove that $a$ and $b_{\theta}$ are all zero for all $\theta \in \Theta$.

First of all we consider the coefficient $a$. A direct calculation shows that

$$
\begin{aligned}
a\left[\sum_{i=1}^{n} x_{i} y^{\lambda} D_{i}, D_{H}\left(x^{u} y^{\eta}\right)\right] & =\sum_{i, j=1}^{n}(-1)^{|u|} a\left[x_{i} y^{\lambda} D_{i}, D_{j}\left(x^{u}\right) y^{\eta} D_{j}\right] \\
& =\sum_{i, j=1}^{n}(-1)^{|u|} a\left[x_{i} y^{\lambda} D_{i},(-1)^{\tau(u, j)} x^{u-\langle j\rangle} y^{\eta} D_{j}\right] \\
& = \begin{cases}-\sum_{j=1}^{n}(-1)^{|u|+\tau(u, j)} a x^{u-\langle j\rangle} y^{\lambda+\eta} D_{j} & \text { if } i=j, \\
(n-1) \sum_{j=1}^{n}(-1)^{|u|+\tau(u, j)} a x^{u-\langle j\rangle} y^{\lambda+\eta} D_{j} & \text { if } i \neq j .\end{cases}
\end{aligned}
$$

Using the similar discussion in Theorem 4.4, we obtain

$$
\operatorname{dim}\left(\operatorname{span}_{\mathbb{F}}\left\{x^{u-\langle j\rangle} y^{\lambda+\eta} D_{j}\right\}\right)>n p^{m}
$$

for given $j \in u$. Since $n>1$, we have $a=0$.

Secondly, the other coefficient $b_{\theta}$ will be considered. For any $u \in \mathbb{B}(n)$ and $\eta \in G$, we have

$$
\begin{aligned}
b_{\theta} D_{\theta}\left(D_{H}\left(x^{u} y^{\eta}\right)\right) & =\tilde{\theta}(\eta) D_{H}\left(b_{\theta} x^{u} y^{\eta}\right) \\
& =\sum_{i=1}^{n}(-1)^{|u|} b_{\theta} D_{i}\left(x^{u}\right) D_{i} \tilde{\theta}(\eta) y^{\eta} \\
& =b_{\theta} \sum_{i=1}^{n}(-1)^{|u|+\tau(u, i)} x^{u-\langle i\rangle} D_{i} \tilde{\theta}(\eta) y^{\eta} .
\end{aligned}
$$

By the equality above and the similar discussion in Theorem 4.4, we have

$$
\operatorname{dim}\left(\operatorname{span}_{\mathbb{F}}\left\{x^{u-\langle i\rangle} \tilde{\eta}(\mu) y^{\eta} D_{j}\right\}\right)>n p^{m} .
$$

Hence $b_{\theta}=0$ for all $\theta \in \Theta$. Therefore, $D=\operatorname{ad} D_{H}(g)$. It follows from Theorem 4.4 that $I(\operatorname{hg}(\operatorname{Der} H))=n p^{m}$. In particular, $I(D)=n p^{m}$ if and only if

$$
0 \neq D \in \operatorname{span}_{\mathbb{F}}\left\{\operatorname{ad} D_{H}\left(x^{\pi} y^{\lambda}\right) \mid \lambda \in G\right\} .
$$


We adopt the notations $n^{\prime}, m^{\prime}$ in Section 3 and let $H^{\prime}=H\left(n^{\prime}, m^{\prime}\right)$ and $G^{\prime}=$ $\left\{\sum_{i=1}^{m^{\prime}} \lambda_{i} z_{i} \mid \lambda_{i} \in \Pi, i=1, \ldots, m^{\prime}\right\}$.

Proposition 4.6. Let

$$
\begin{aligned}
R & =\operatorname{span}_{\mathbb{F}}\left\{D_{H}\left(x^{u} y^{\lambda}\right) \in H|u \in \mathbb{B}(n),| u \mid \geq 2, \lambda \in G\right\}, \\
R^{\prime} & =\operatorname{span}_{\mathbb{F}}\left\{D_{H^{\prime}}\left(x^{u} y^{\lambda}\right) \in H^{\prime}\left|u \in \mathbb{B}\left(n^{\prime}\right),\right| u \mid \geq 2, \lambda \in G^{\prime}\right\} .
\end{aligned}
$$

If $\sigma$ is an isomorphism from $H$ to $H^{\prime}$, then $\sigma(R)=R^{\prime}$.

Proof. It is easy to see that the map $\xi: D \rightarrow \sigma D \sigma^{-1}$ is a bijection. Then $\xi$ is an isomorphism from Der $H$ to Der $H^{\prime}$. Thus $I(\operatorname{hg}(\operatorname{Der} H))=I\left(\operatorname{hg}\left(\operatorname{Der} H^{\prime}\right)\right)$. According to Theorem 4.5, we have

$$
\sigma\left(\operatorname{span}_{\mathbb{F}}\left\{\operatorname{ad} D_{H}\left(x^{\pi} y^{\lambda}\right)\right\}\right) \sigma^{-1}=\operatorname{span}_{\mathbb{F}}\left\{\operatorname{ad} D_{H}\left(x^{\pi^{\prime}} y^{\lambda^{\prime}}\right)\right\},
$$

where $\pi^{\prime}=\left\{1, \ldots, n^{\prime}\right\} \in \mathbb{B}\left(n^{\prime}\right), \lambda \in G$, and $\lambda^{\prime} \in G^{\prime}$. Note that

$$
\left[D_{H}\left(x^{\pi} y^{\lambda}\right), D_{H}\left(x^{u} y^{\eta}\right)\right]=D_{H}\left(\sum_{i=1}^{n}(-1)^{n} D_{i}\left(x^{\pi} y^{\lambda}\right) D_{i}\left(x^{u} y^{\eta}\right)\right) .
$$

for $u \in \mathbb{B}(n)$ and $\lambda, \eta \in G$. If $|u| \geq 2$, then $D_{i}\left(x^{u} y^{\eta}\right)=(-1)^{\tau(u, i)} x^{u-\langle i\rangle} y^{\eta}$ and $D_{i}\left(x^{\pi} y^{\lambda}\right)=(-1)^{\tau(\pi, i)} x^{\pi-\langle i\rangle} y^{\lambda}$. Since $\{u-\langle i\rangle\} \in\{\pi-\langle i\rangle\}$, we have

$$
\left[D_{H}\left(x^{\pi} y^{\lambda}\right), D_{H}\left(x^{u} y^{\eta}\right)\right]=0 \text {. }
$$

Hence

$$
R=\left\{h \in H \mid\left(\operatorname{span}_{\mathbb{F}}\left\{\operatorname{ad} D_{H}\left(x^{\pi} y^{\lambda}\right)\right\}\right)(h)=0\right\} .
$$

Similarly, $R^{\prime}=\left\{h \in H^{\prime} \mid\left(\operatorname{span}_{\mathbb{F}}\left\{\operatorname{ad} D_{H^{\prime}}\left(x^{\pi^{\prime}} y^{\lambda^{\prime}}\right)\right\}\right)(h)=0\right\}$. Then

$$
\begin{aligned}
\left(\operatorname{span}_{\mathbb{F}}\left\{\operatorname{ad} D_{H^{\prime}}\left(x^{\pi^{\prime}} y^{\lambda^{\prime}}\right)\right\}\right)(\sigma(R)) & =\sigma\left(\operatorname{span}_{\mathbb{F}}\left\{\operatorname{ad} D_{H}\left(x^{\pi} y^{\lambda}\right)\right\}\right) \sigma^{-1}(\sigma(R)) \\
& =\sigma\left(\operatorname{span}_{\mathbb{F}}\left\{\operatorname{ad} D_{H}\left(x^{\pi} y^{\lambda}\right)\right\}\right)(R) \\
& =\sigma\left(\operatorname{span}_{\mathbb{F}}\left\{\operatorname{ad} D_{H}\left(x^{\pi} y^{\lambda}\right)\right\}\right)(R) \\
& =\sigma(0) \\
& =0 .
\end{aligned}
$$

Thus $\sigma(R) \subseteq R^{\prime}$. By the same method above, we have $\sigma^{-1}\left(R^{\prime}\right) \subseteq R$. Hence $R^{\prime} \subseteq \sigma(R)$. In conclusion, $\sigma(R)=R^{\prime}$.

Lemma 4.7. Let $H=H_{(-1)} \supseteq H_{(0)} \supseteq \cdots \supseteq H_{(n-3)} \supseteq H_{(n-2)}=0$ be the natural filtration of $H$. Then

$$
H_{(0)}=R, \quad H_{(i)}=\left\{h \in H_{(i-1)} \mid[h, H] \subseteq H_{(i-1)}\right\} \text { for } i \geq 1 .
$$


Similarly, for the natural filtration of $H^{\prime}$,

$$
H_{(0)}^{\prime}=R^{\prime}, \quad H_{(i)}^{\prime}=\left\{h \in H_{(i-1)}^{\prime} \mid\left[h, H^{\prime}\right] \subseteq H_{(i-1)}^{\prime}\right\} \text { for } i \geq 1 .
$$

Proof. Suppose that $M=\left\{h \in H_{(i-1)} \mid[h, H] \subseteq H_{(i-1)}\right\}$. Note that $H_{(i)} \subseteq H_{(i-1)}$ and $\left[H_{(i)}, H\right]=\left[H_{(i)}, H_{(-1)}\right] \subseteq H_{(i-1)}$. Then the inclusion relations show that $H_{(i)} \subseteq M$.

Conversely, if $h \in M$, then $h \in H_{(i-1)}$. So we may suppose that $h=\sum_{j=i-1}^{n-3} h_{j}$, where $h_{j} \in H_{j}$. Let $h_{i-1}=\sum_{k} a_{k} D\left(x^{u_{k}} y^{\lambda_{k}}\right)$, where $a_{k} \in \mathbb{F}, u_{k} \in \mathbb{B}(n),\left|u_{k}\right|=$ $i-1+2=i+1 \geq 2$, and $\lambda_{k} \in G$.

If $h_{i-1}=0$, then $h \in H_{(i)}$. Therefore, the desired result follows in this case.

If $h_{i-1} \neq 0$, then it follows from $h \in M$ that $\left[h, H_{-1}\right] \subseteq H_{(i-1)}$. Hence $\left[h_{i-1}, H_{-1}\right]=0$, that is,

$$
\left[\sum_{k} a_{k} D\left(x^{u_{k}} y^{\lambda_{k}}\right), D_{H}\left(x_{i} y^{\eta}\right)\right]=0
$$

for all $i \in \mathrm{Y}$ and $\eta \in G$. As $\left|u_{k}\right| \geq 2$, there exists $i \in \mathrm{Y}$ such that

$$
D_{H}\left((-1)^{\left|u_{k}\right|} D_{i}\left(x^{u_{k}} y^{\lambda_{k}}\right)\right) \neq 0 \text {. }
$$

Hence $a_{k}=0$ which is in contradiction with $h_{i-1} \neq 0$.

The considerations above show that $M \subseteq H_{(i)}$. Therefore,

$$
H_{(i)}=\left\{h \in H_{(i-1)} \mid[h, H] \subseteq H_{(i-1)}\right\} \quad \text { for } i \geq 1 .
$$

Similarly, $H_{(i)}^{\prime}=\left\{h \in H_{(i-1)}^{\prime} \mid\left[h, H^{\prime}\right] \subseteq H_{(i-1)}^{\prime}\right\}$ for $i \geq 1$.

Proposition 4.8. Suppose that $H \cong H^{\prime}$ and $\sigma$ is an isomorphism from $H$ to $H^{\prime}$, then $\sigma\left(H_{(i)}\right)=H_{(i)}^{\prime}$ for all $i \geq-1$.

Proof. If $i=0$, then $H_{(0)}=R$ and $H_{(0)}^{\prime}=R^{\prime}$. Proposition 4.6 shows that $\sigma\left(H_{(0)}\right)=H_{(0)}^{\prime}$.

If $i=-1$, then $H_{(-1)}=H$ and $H_{(-1)}^{\prime}=H^{\prime}$. Hence $\sigma\left(H_{(-1)}\right)=H_{(-1)}^{\prime}$.

Next we use induction on $i$. Assume that $\sigma\left(H_{(i)}\right)=H_{(i)}^{\prime}$ for $i \geq 1$. By Lemma 4.7, for $h \in H_{(i+1)}$, we have $h \in H_{(i)}$ as well as $[h, H] \subseteq H_{(i)}$. Since $h \in H_{(i)}$, the induction hypothesis yields $\sigma(h) \in H_{(i)}^{\prime}$. Then

$$
\sigma([h, H])=[\sigma(h), \sigma(H)] \subseteq\left[H_{(i)}^{\prime}, H^{\prime}\right] \subseteq H_{(i)}^{\prime} .
$$

By Lemma 4.7, we have $\sigma(h) \in H_{(i+1)}^{\prime}$. This implies that $\sigma\left(H_{(i+1)}\right) \subseteq H_{(i+1)}^{\prime}$.

For any $h^{\prime} \in H_{(i+1)}^{\prime}$, we want to prove that $h^{\prime} \in \sigma\left(H_{(i+1)}\right)$. The fact $h^{\prime} \in H_{(i)}^{\prime}=$ $\sigma\left(H_{(i)}\right)$ ensures that there exists $h \in H_{(i)}$ such that $\sigma(h)=h^{\prime}$. It is easy to see that $\left[h^{\prime}, H^{\prime}\right] \subseteq H_{(i)}^{\prime}=\sigma\left(H_{(i)}\right)$. Since $\left[h^{\prime}, H^{\prime}\right]=[\sigma(h), \sigma(H)]=\sigma[h, H]$, we have $[h, H] \in H_{(i)}$. Then $h \in H_{(i+1)}$, that is, $h^{\prime} \in \sigma\left(H_{(i+1)}\right)$. Consequently, $\sigma\left(H_{(i)}\right)=H_{(i)}^{\prime}$ for all $i \geq-1$. 
Theorem 4.9. The natural filtration of $H$ is invariant under the automorphisms of $H$.

Proof. It is a direct conclusion of Proposition 4.8.

Imitating the definition of $\mathfrak{W}_{i}$ in $W$, we let $\mathfrak{H}_{i}=H_{(i)} / H_{(i+1)}$ for $-1 \leq i \leq n-3$. Suppose that $\mathfrak{H}:=\bigoplus_{i=-1}^{n-3} \mathfrak{H}_{i}$, then $\mathfrak{H}$ is a $\mathbb{Z}$-graded space. Let $x+H_{(i+1)} \in \mathfrak{H}_{i}$ and $y+H_{(j+1)} \in \mathfrak{H}_{j}$. We define

$$
\left[x+H_{(i+1)}, y+H_{(j+1)}\right]:=[x, y]+H_{(i+j+1)} .
$$

It is easy to see that the operator [, ] on $\mathfrak{H}$ is well-defined. There exists a linear expansion such that $\mathfrak{H}$ has a operator [ , ]. A direct verification shows that $\mathfrak{H}$ is a Lie superalgebra with respect to the operator [ , ]. The Lie superalgebras $\mathfrak{H}$ is called a Lie superalgebra induced by the natural filtration of $H$.

By the similar methods used to prove Propositions 3.7 and 3.9, the following lemmas are easy to obtain.

Lemma 4.10. $\mathfrak{H} \cong H$.

Lemma 4.11. Suppose that $H \cong H^{\prime}$ and $\sigma$ is an isomorphism from $H$ to $H^{\prime}$, then $\sigma$ induces an isomorphism $\tilde{\sigma}$ from $\mathfrak{H}$ to $\mathfrak{H}^{\prime}$ such that $\tilde{\sigma}\left(\mathfrak{H}_{i}\right)=\mathfrak{H}_{i}^{\prime}$ for all $i \geq-1$.

Theorem 4.12. $H \cong H^{\prime}$ if and only if $m=m^{\prime}$ and $n=n^{\prime}$.

Proof. Since the sufficiency is obvious, it suffices to prove the necessity. Using the similar methods in the proof of Theorem 3.10, we have $\operatorname{dim} H_{-1}=\operatorname{dim} H_{-1}^{\prime}$ and $\operatorname{dim} H_{0}=\operatorname{dim} H_{0}^{\prime}$. It follows from $W_{-1}=H_{-1}$ that $n p^{m}=n^{\prime} p^{m^{\prime}}$. By virtue of the definition of $H_{i}$, we have

$$
H_{0}=\operatorname{span}_{\mathbb{F}}\left\{D_{H}\left(x_{i} x_{j} y^{\lambda}\right) \in H \mid i, j \in \mathrm{Y}, \lambda \in G\right\} .
$$

Thus $\operatorname{dim} H_{0}=\mathrm{C}_{n}^{2} p^{m}=\frac{1}{2} n(n-1) p^{m}$. Similarly, $\operatorname{dim} H_{0}^{\prime}=\frac{1}{2} n^{\prime}\left(n^{\prime}-1\right) p^{m^{\prime}}$. According to $\operatorname{dim} H_{0}=\operatorname{dim} H_{0}^{\prime}$ and $n p^{m}=n^{\prime} p^{m^{\prime}}$, we have $n=n^{\prime}$ and $m=m^{\prime}$. Consequently, the desired result follows.

\section{Acknowledgments}

The authors thank Professors Liangyun Chen, Yang Jiang and Ruimei Gao for their helpful comments and suggestions. We also give our special thanks to referees for many helpful suggestions.

\section{References}

[Awuti and Zhang 2008] S. Awuti and Y. Z. Zhang, "Modular Lie superalgebra $\bar{W}(n, m)$ ", Dongbei Shida Xuebao 40:2 (2008), 7-11. MR 2009k:17035 Zbl 1171.17302

[Block and Wilson 1988] R. E. Block and R. L. Wilson, "Classification of the restricted simple Lie algebras”, J. Algebra 114:1 (1988), 115-259. MR 89e:17014 Zbl 0644.17008 
[Bouarroudj and Leites 2006] S. Bouarroudj and D. Leites, "Simple Lie superalgebras and nonintegrable distributions in characteristic p", Zap. Nauchn. Sem. S.-Peterburg. Otdel. Mat. Inst. Steklov. (POMI) 331:14 (2006), 15-29. In Russian; translated in J. Math. Sci. 141:4 (2007), 1390-1398. MR 2007d:17025 Zbl 1104.17011

[Chen and Liu 2011] Y. Chen and W. Liu, "Finite-dimensional odd contact superalgebras over a field of prime characteristic", J. Lie Theory 21:3 (2011), 729-754. MR 2012h:17024 Zbl 1239.17015

[Elduque 2007] A. Elduque, "Some new simple modular Lie superalgebras", Pacific J. Math. 231:2 (2007), 337-359. MR 2009d:17027 Zbl 1207.17023

[Jacobson 1962] N. Jacobson, Lie algebras, Interscience Tracts in Pure and Applied Mathematics 10, Interscience, New York, 1962. MR 26 \#1345 Zbl 0121.27504

[Jin 1992] N. Jin, "Ad-nilpotent elements, quasi-nilpotent elements and invariant filtrations of infinitedimensional Lie algebras of Cartan type", Sci. China Ser. A 35:10 (1992), 1191-1200. MR 94j:17027 Zbl 0772.17007

[Kac 1974] V. G. Kac, "A description of the filtered Lie algebras with which graded Lie algebras of Cartan type are associated", Izv. Akad. Nauk SSSR Ser. Mat. 38 (1974), 800-834. In Russian; translated in Math. USSR Izv 8:4 (1974), 801-835. MR 51 \#5685 Zbl 0317.17002

[Kac 1977] V. G. Kac, "Lie superalgebras”, Advances in Math. 26:1 (1977), 8-96. MR 58 \#5803 Zbl 0366.17012

[Kochetkov and Leites 1992] Y. Kochetkov and D. Leites, "Simple Lie algebras in characteristic 2 recovered from superalgebras and on the notion of a simple finite group", pp. 59-67 in Proceedings of the International Conference on Algebra, II (Novosibirsk, 1989), edited by L. A. Bokut et al., Contemp. Math. 131, Amer. Math. Soc., Providence, RI, 1992. MR 93g:17035 Zbl 0765.17006

[Kostrikin and Shafarevich 1969] A. I. Kostrikin and I. R. Shafarevich, "Graded Lie algebras of finite characteristic", Izv. Akad. Nauk SSSR Ser. Mat. 33 (1969), 251-322. In Russian; translated in Math. USSR Izv 3:2 (1969), 237-304. MR 40 \#5680 Zbl 0211.05304

[Petrogradski 1992] V. M. Petrogradski, "Identities in the enveloping algebras for modular Lie superalgebras", J. Algebra 145:1 (1992), 1-21. MR 92m:17013 Zbl 0752.17001

[Ren et al. 2011] L. Ren, Q. Mu, and Y. Zhang, "A class of finite-dimensional Lie superalgebra of Hamiltonian type", Algebra Colloq. 18:2 (2011), 347-360. MR 2012g:17025 Zbl 1219.17015

[Ren et al. 2012] L. Ren, Q. Mu, and Y. Zhang, "Odd Hamiltonian superalgebras and special odd Hamiltonian superalgebras of formal vector fields", Pacific J. Math. 257:2 (2012), 471-489. MR 2972474 Zbl 06080829

[Scheunert 1979] M. Scheunert, The theory of Lie superalgebras: An introduction, Lecture Notes in Mathematics 716, Springer, Berlin, 1979. MR 80i:17005 Zbl 0407.17001

[Strade 1993] H. Strade, "The classification of the simple modular Lie algebras, IV: Determining the associated graded algebra”, Ann. of Math. (2) 138:1 (1993), 1-59. MR 94k:17039 Zbl 0790.17011

[Varadarajan 2004] V. S. Varadarajan, Supersymmetry for mathematicians: An introduction, Courant Lecture Notes in Mathematics 11, Amer. Math. Soc., Providence, RI, 2004. MR 2005g:58011 Zbl 1142.58009

[Yuan et al. 2011] J. Yuan, Y. Chen, and W. Liu, "Automorphisms and p-characters of restricted Lie superalgebras of Cartan type”, Algebra Colloq. 18:3 (2011), 397-410. MR 2012g:17027 Zbl 1231.17010

[Zhang 1997] Y. Zhang, "Finite-dimensional Lie superalgebras of Cartan type over fields of prime characteristic", Chinese Sci. Bull. 42:9 (1997), 720-724. MR 98h:17023 Zbl 0886.17022

[Zhang and Fu 2002] Y. Zhang and H. Fu, "Finite-dimensional Hamiltonian Lie superalgebra", Comm. Algebra 30:6 (2002), 2651-2673. MR 2003h:17040 Zbl 1021.17017 
[Zhang and Nan 1998] Y. Zhang and J. Nan, "Finite-dimensional Lie superalgebras $W(m, n, \mathbf{t})$ and $S(m, n, \mathbf{t})$ of Cartan type", Adv. Math. (China) 27:3 (1998), 240-246. MR 99h:17012 Zbl 1054.17502 [Zhao 2010] L. Zhao, "Representations of Lie superalgebras in prime characteristic, III", Pacific J. Math. 248:2 (2010), 493-510. MR 2012d:17037 Zbl 1225.17022

Received December 17, 2012. Revised August 27, 2013.

\section{KELI ZHENG}

SCHOOL OF MATHEMATICS AND STATISTICS

NORTHEAST NORMAL UNIVERSITY

ChangChun, 130024

CHINA

Current address:

DEPARTMENT OF MATHEMATICS

NORTHEAST FORESTRY UNIVERSITY

HARBIN 150040

CHINA

zhengk1561@nenu.edu.cn

YONGZHENG ZHANG

SCHOOL OF MATHEMATICS AND STATISTICS

NORTHEAST NORMAL UNIVERSITY

ChangChUn, 130024

CHINA

zhyz@nenu.edu.cn

WEI SONG

DongGuAN MiddLE SCHOOL - SSL SCHOOL

DONGGUAN, 523808

CHINA

songw207@163.com 


\title{
PACIFIC JOURNAL OF MATHEMATICS
}

\author{
msp.org/pjm
}

Founded in 1951 by E. F. Beckenbach (1906-1982) and F. Wolf (1904-1989)

\section{EDITORS}

Don Blasius (Managing Editor)

Department of Mathematics

University of California

Los Angeles, CA 90095-1555

blasius@math.ucla.edu

\author{
Paul Balmer \\ Department of Mathematics \\ University of California \\ Los Angeles, CA 90095-1555 \\ balmer@math.ucla.edu \\ Robert Finn \\ Department of Mathematics \\ Stanford University \\ Stanford, CA 94305-2125 \\ finn@math.stanford.edu \\ Sorin Popa \\ Department of Mathematics \\ University of California \\ Los Angeles, CA 90095-1555 \\ popa@math.ucla.edu
}

\author{
Vyjayanthi Chari \\ Department of Mathematics \\ University of California \\ Riverside, CA 92521-0135 \\ chari@math.ucr.edu \\ Kefeng Liu \\ Department of Mathematics \\ University of California \\ Los Angeles, CA 90095-1555 \\ liu@math.ucla.edu \\ Jie Qing \\ Department of Mathematics \\ University of California \\ Santa Cruz, CA 95064 \\ qing@ cats.ucsc.edu
}

\section{PRODUCTION}

Silvio Levy, Scientific Editor, production@msp.org

\section{SUPPORTING INSTITUTIONS}

ACADEMIA SINICA, TAIPEI

CALIFORNIA INST. OF TECHNOLOGY

INST. DE MATEMÁTICA PURA E APLICADA

KEIO UNIVERSITY

MATH. SCIENCES RESEARCH INSTITUTE

NEW MEXICO STATE UNIV.

OREGON STATE UNIV.

\author{
STANFORD UNIVERSITY \\ UNIV. OF BRITISH COLUMBIA \\ UNIV. OF CALIFORNIA, BERKELEY \\ UNIV. OF CALIFORNIA, DAVIS \\ UNIV. OF CALIFORNIA, LOS ANGELES \\ UNIV. OF CALIFORNIA, RIVERSIDE \\ UNIV. OF CALIFORNIA, SAN DIEGO \\ UNIV. OF CALIF., SANTA BARBARA
}

\author{
Daryl Cooper \\ Department of Mathematics \\ University of California \\ Santa Barbara, CA 93106-3080 \\ cooper@math.ucsb.edu \\ Jiang-Hua Lu \\ Department of Mathematics \\ The University of Hong Kong \\ Pokfulam Rd., Hong Kong \\ jhlu@maths.hku.hk \\ Paul Yang \\ Department of Mathematics \\ Princeton University \\ Princeton NJ 08544-1000 \\ yang@math.princeton.edu
}

These supporting institutions contribute to the cost of publication of this Journal, but they are not owners or publishers and have no responsibility for its contents or policies.

See inside back cover or msp.org/pjm for submission instructions.

The subscription price for 2014 is US $\$ 410 /$ year for the electronic version, and \$535/year for print and electronic.

Subscriptions, requests for back issues and changes of subscribers address should be sent to Pacific Journal of Mathematics, P.O. Box 4163, Berkeley, CA 94704-0163, U.S.A. The Pacific Journal of Mathematics is indexed by Mathematical Reviews, Zentralblatt MATH, PASCAL CNRS Index, Referativnyi Zhurnal, Current Mathematical Publications and Web of Knowledge (Science Citation Index).

The Pacific Journal of Mathematics (ISSN 0030-8730) at the University of California, c/o Department of Mathematics, 798 Evans Hall \#3840, Berkeley, CA 94720-3840, is published twelve times a year. Periodical rate postage paid at Berkeley, CA 94704, and additional mailing offices. POSTMASTER: send address changes to Pacific Journal of Mathematics, P.O. Box 4163, Berkeley, CA 94704-0163.

PJM peer review and production are managed by EditFLOW ${ }^{\circledR}$ from Mathematical Sciences Publishers.

\section{PUBLISHED BY}

\section{mathematical sciences publishers \\ nonprofit scientific publishing}

http://msp.org/

(C) 2014 Mathematical Sciences Publishers 


\section{PACIFIC JOURNAL OF MATHEMATICS}

Volume 269 No. $1 \quad$ May 2014

The asymptotic behavior of Palais-Smale sequences on manifolds with $\quad 1$ boundary

SÉRGIO AlMARAZ

The cup subalgebra of a $\mathrm{II}_{1}$ factor given by a subfactor planar algebra is maximal amenable

ARNAUD BROTHIER

Representation theory of type B and C standard Levi $W$-algebras

JONATHAN BROWN and SIMON M. GOODWIN

New invariants for complex manifolds and rational singularities

RONG DU and YUN GAO

Homogeneity groups of ends of open 3-manifolds

DENNIS J. GARITY and DUŠAN REPOVŠ

On the concircular curvature of a $(\kappa, \mu, \nu)$-manifold

Florence Gouli-Andreou and Evaggelia Moutafi

Genuses of cluster quivers of finite mutation type

FANG LI, JICHUN LIU and YICHAO YANG

Taut foliations in knot complements

TAO LI and RACHEL ROBERTS

On the set of maximal nilpotent supports of supercuspidal representations 169

QIN YUJUN

The natural filtrations of finite-dimensional modular Lie superalgebras of 199 Witt and Hamiltonian type

Keli Zheng, Yongzheng ZHANG and WeI Song

Free Brownian motion and free convolution semigroups: multiplicative 219 case

PING ZHONG 